\title{
A INCIDÊNCIA DE CIRURGIAS NA POPULAÇÃO DE RIBEIRÃO PRETO, SP, BRASIL *
}

Marilisa Berti de Azevedo Barros **

BARROS, M. B. de A. A incidencia de cirurgias na população de Ribeirão Preto,

SP, Brasil. Rev, Saúde vúbl., S. Paulo, 16:268-81, 1982.

RESUMO: Foi estudada a incidência de cirurgias na população residente em Ribeirão Preto, São Paulo (Brasil), em 1975, em relação à idade, sexo, categoria de internação do paciente e tipo de internação cirúrgica, utilizando-se as informações coletadas por um Centro de Processamento de Dados Hospitalares. Foram observadas elevadas taxas de cirurgias: $79,8 / 1000$ no sexo feminino e $43,8 / 1000$ no masculino. As intervenções obstétricas representaram $31,8 \%$ do total de operações realizadas; no sexo masculino as intervençôes ortopédicas foram as de maior incidência. A proporção de internações com ocorrência de cirurgia foi mais elevada nos pacientes particulares. As operaçóes ortopédicas e plásticas incidiram relativamente mais nos beneciários da Previdência Social e nos indigentes, enquanto que as otorrinológicas e urológicas foram proporcionalmenta mais frequentes nos pacientes particulares.

UNITERMOS: Cirurgias, incidência. Hospitalização, Ribeirão Preto, SP.

\section{INTRODUÇAOO}

Estudos desenvolvidos em vários paises têm revelado ampla variação na ocorrência de intervenções cirúrgicas.

Glover, (citado por Lewis ${ }^{10}$, 1969) já em 1938, observou variações surpreendentes nas taxas de tonsilectomia entre várias regiões da Inglaterra.

Lembcke 9 (1952), estudando a ocorrència de apendicectomia em áreas do estado de Nova York, verificou oscilação de 2,9 a 7,1 (por 1.000 habitantes), sendo que nos locais onde a cirurgia era menos freqüente não se elevava a mortalidade por apendicite.
Várias cirurgias apresentaram incidência diversa nas regiões da Inglaterra, tendo Logan e Eimerl ${ }^{11}$ (1965) atribuído relevância ao papel de médico geral, encaminhando ou não o paciente para a intervenção.

Bunker ${ }^{4}$ (1970) verificou que a taxa de cirurgias assim como o número de cirurgiões por habitantes, na Inglaterra, equivaliam à metade dos valores encontrados nos EUA, em período de tempo correspondente.

Pearson e col.1i (1968) encontraram taxa global de intervençñes cirúrgicas quase duas

* Este estudo é parte da Dissertação de Mestrado apresentada pela autora à Faculdade de Medicina de Ribeirão Preto, USP, em 1977: "Estudo da Morbidade Hospitalar no Município de Ribeirão Preto, em 1975", desenvolvida com auxilio finarceiro da FAPESP.

* Do Departamento de Medicina Preventiva e Social da Faculdade de Ciências Médicas da Universidade Estatual de Campinas - Rua Dr. Quirino, 1856 - Centro - 13100 - Campinas, SP Brasil. 
EARROS, M.B. de A. A incidência de cirurgias na população de Ribeirão Preto, SP, Brasil. Rev. Saúde puibl., S. Paulo, 16:268-81, 1982.

vezes maior em New England (EUA) que em Liverpool (Inglaterra). A taxa de tonsilectomia e/ou adenoidectomia da população de New England foi quatro vezes superior à observada em Uppsala (Suécia).

Lewis 10 (1969) verificou diferenças de até quatro vezes na ocorrência de seis procedimentos cirúrgicos entre regióes do estado de Kansas.

Os dados revelam, no geral, correlação positiva entre taxas de cirurgias e de internações.

Foi observado que a freqüência de intervenções cirúrgicas no Canadá era quase duas vezes superior à do Reino Unido, sendo que, no primeiro, as taxas de colecistectomia eram cinco vezes maior, enquanto que a mortalidade por patologias da vesícula biliar era o dobro daquela verificada para Inglaterra e Gales (Vayda ${ }^{23}$, 1973). Entre 49 condados de Ontario, Stockwell e Vayda ${ }^{21}$ (1979), encontraram variações de 5 a 8 vezes na freqüência de tonsilectomia, colectomia, histerectomia, colecistectomia e apendicectomia. Também têm sido verificadas diferentes taxas entre estratos sociais da mesma comunidade.

Crianças cujos pais têm ocupação de melhor nivel são mais submetidas a tonsilectomias e postectomias que aquelas de familias com ocupações menos qualificadas (Bolande 3 , 1969). Referindo-se a cuidados médicos em geral, White ${ }^{26}$ (1968) observou que nos EUA a utilização de serviços aumenta com a renda familiar. Salkewer 20 (1975), analisando dados de vários países europeus, observou correlação positiva entre uso de cuidados médicos e nível sócio-econômico, especialmente na população infantil.

Ao analisar essas diferenças encontradas entre paises, entre regiōes de um mesmo pais ou ainda entre grupos sócio-econômicos discriminados de uma mesma população, alguns autores, embora reconhecendo diferenças nos padrões de morbidade, têm atribuido grande parcela das diferenças verificadas à disponibilidade de recursos médico-hospitalares. Vayda ${ }^{23}$ (1973) enfatiza que "entre os fatores que contribuem estão os diferentes estilos de tratamento e critérios de indicação de cirugia, mas os principais determinantes podem ser as diferenças na organização e pagamento dos serviços de saúde, as limitações de credenciamentos para atos cirúrgicos e o número de cirurgiões e leitos hospitalares". Várias são as observações que reforçam essa assertiva de Vayda. Lewis ${ }^{10}$ (1969) verificou por meio de análise de regressão múltipla que o número de cirurgias tinha como bons preditores o número de leitos hospitalares e o tiúmero de cirurgiões. Stockwell e Vayda ${ }^{21}$ (1979) referem que $50 \%$ da variação nas taxas que encontraram era explicada pelo indice de leitos e médicos. Perrot ${ }^{1 s}$ (1966) observou que os empregados federais dos EUA, cobertos por planos de pré-pagamento, apresentavam as menores taxas de cirurgias. Diferenças importantes nas taxas de intervenções cirúrgicas eletivas têm sido observadas em pessoas sujeitas a diferentes modalidades de pagamento da assistência médica, como assinalam Logerfo e col.13 (1979) que encontraram $43 \%$ de cirurgias "desnecessárias" em um plano com pagamento por serviço (IPP) e $22 \%$ em plano de medicina de grupo com pré-pagamento (PGP).

As diferenças nas taxas de utilização de serviços de saúde têm seu interesse ressaltado porque concorrem com gastos crescentes no orçamento dos países, sem que se verifique, quando se consideram os paises desenvolvidos, correlação entre os indicadores de saúde e a quantidade de recursos aplicados (Peterson e col.19, 1967). Por outro lado, as cirurgias representam o cuidado médico de maior custo implicando quase sempre hospitalização do paciente, além do fato de que incorporam grande parte do avanço tecnológico que se dá na área médica.

As pesquisas sobre utilização de serviços médicos são numerosas nos paises desenvolvidos (Anderson e Andersen 1, 1972), de sorte que um conjunto apreciável de infor- 
EÁRROS, M.B. de A. A incidência de cirurgias na população de Ribeirão Preto, SP, Brasil. Rev. Saúde públ., S. Paulo, 16:268-81, 1982.

mações encontra-se disponivel para análises mais detalhadas e inclusive para testar algumas hipóteses.

$\mathrm{Na}$ realidade brasileira, entretanto, escasseiam as investigações empíricas sobre o consumo de serviços. Parte das dificuldades existentes para a realização desses estudos deve-se à problemática caracterização da natureza da demanda ao nível das instituições médicas, que esbarra na má qualidade dos registros médicos existentes; além disso, as instituiçōes (ambulatórios, hospitais, e outros) não têm, em geral, uma população de referência, geograficamente bem definida, o que impossibilita a elaboração de taxas de consumo. Por cutro lado, pesquisas que tomam o consumidor como unidade de investigação implicam uma quantidade de recursos raramente disponível entre nós.

Tendo em vista a importância do tema nas questões relativas à saúde e a carência de pesquisas que revelem a intensidade e natureza do consumo de atos médicos, em localidades brasileiras, propusemo-nos a desenvolver um estudo descritivo das cirurgias ocorridas na população residente em Ribeirão Preto, Estado de São Paulo. Acreditamos que a descrição de situações e diferenciações concretas ainda que restritas, poderiam contribuir para a compreensão da prática médica, ao oferecer subsídios para análises mais amplas.

O estudo foi possibilitado pela existência de um Centro de Processamento de Dados Hospitalares (CPDH), cujas informações, consideradas de boa qualidade por Yazille Rocha ${ }^{24}$ (1975), permitiu-nos selecionar as intervenções cirúrgicas realizadas nos residentes de Ribeirão Preto. As variáveis incluidas no estudo restringiram-se, portanto, às existentes na folha de alta hospitalar do CPDH.

O objetivo deste trabalho é caracterizar a incidência de cirurgias segundo idade, sexo e categoria de internação do paciente, assim como o tipo de cirurgias realizadas. Procura-se, de uma maneira ainda que preliminar, detectar diferenciaçōes no perfil de cirurgias das diferentes modalidades de atenção médica.

\section{MATERIAL E METODOS}

Foram estudadas as altas hospitalares de pacientes submetidos a cirurgias ocorridas nos hospitais de Ribeirão Preto, durante o ano de 1975, referentes às pessoas residentes nesse município.

As informações foram coletadas e processadas pelo CPDH que funciona junto ao Departamento de Medicina Social da Faculdade de Medicina de Ribeirão Preto, cujas atividades foram descritas e analisadas por Fávero e col.8 (1973) e Yazlle Rocha ${ }^{24}$ (1975).

Com referência a cada alta foram estudadas as variáveis: sexo, idade, local de residência, categoria de internação e a principal intervenção cirúrgica realizada.

As cirurgias, que são anotadas no momento da alta do paciente, foram codificadas e tabuladas segundo a "Classificación Internacional de Operaciones Quirurgicas y de Otros Procedimientos Terapeuticos y Diagnosticos" (OPS 16, 1971).

Quanto à categoria de internação, os pacientes foram classificados em: particular - quando a hospitalização foi paga pelo paciente; INPS * (Instituto Nacional de Previdência Social) - quando a internação foi custeada por esta instituição previdênciária; indigente - quando foi gratuito 0 atendimento médico-hospitalar; outro seguro - que incluiu as hospitalizações custeadas pelas demais instituições como UNIMED de Ribeirão Preto - Cooperativa de Trabalho Médico, Instituto de Assistência Médica do Servidor Público Estadual (IAMSPE), entre outras.

A categoria de internação, ao mesmo tempo em que representa as instituições

\footnotetext{
Atual Instituto Nacional de Assistência Médica de Previdência Social (INAMPS).
} 
BARROS, M.B. de A. A incidência de cirurgias na população de Ribeirão Preto, SP, Brasil. Rev. Saude publ., S. Paulo, 16:268-81, 1982.

médicas a que as pessoas podem ter acesso, indicando, de modo imediato, tipos diferentes de seviços de saúde, pode também ser tomada como um indicador de estrato sócio-econômico, pois o tipo de inserção das pessoas no mercado de trabalho define a modalidade de assistência médica que podem obter.

Dado que Ribeirão Preto é um centro regional de assistência médica é possivel esperar que a maior parte das cirurgias realizadas nos residentes no município tenham ocorrido localmente.

\section{RESULTADOS E DISCUSSÃO}

Em 1975, foram realizadas 15.403 cirurgias en pessoas residentes no municipio de Ribeirão Preto, equivalendo a uma taxa de 62,24 intervenções por 1.000 habitantes, sendo de 79,82 no sexo feminino e 43,77 no masculino.

Observa-se na Fig. 1 que a taxa de cirurgias cresce com a idade no sexo masculino, enquanto que no feminino é muito elevada na idade reprodutiva. Se excluidas as cirurgias obstétricas, as mulheres passam

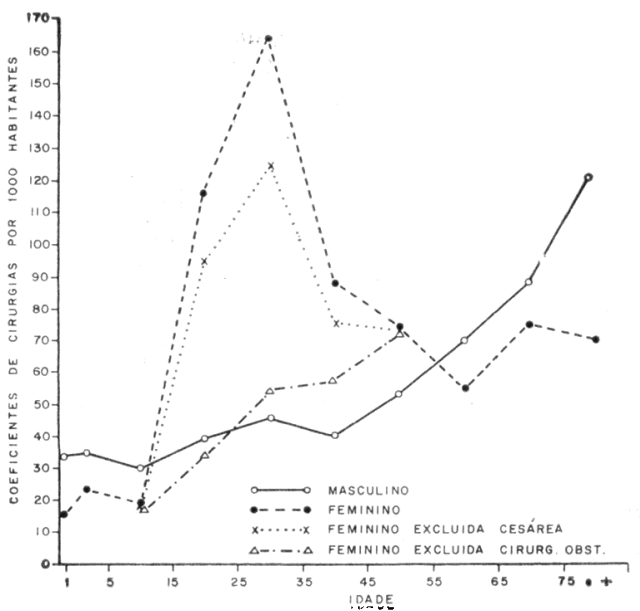

Fig. 1 - Coeficientes de cirurgias de pacientes residentes em Ribeirão Preto, segundo sexo idade. Hospitais Gerais de Ribeirão Preto - 1975. a apresentar taxa global de 41,20, inferior, portanto, à do outro sexo.

A comparação de taxas de cirurgias, entre localidades, restringe-se a informações derivadas de estudos desenvolvidos com objetivos e metodologias diferentes, realizados em contextos distintos quanto à qualidade dos rtgistros médicos e das fontes de dados, quanto à composição etária das populaçōes estudadas, além, de serem realizados em periodos de tempo diferentes. Comparações adequadas exigem estudos internacionais que empreguem métodos $\mathrm{e}$ instrumentos padronizados. (White ${ }^{27}, 1967$ ).

Dessa forma, ao se tomar a incidencia de intervenções cirúrgicas observadas por outros autores ( $T$ abelas 1 e 2), apenas com a finalidade de situar alguns pontos de referência, verifica-se que em Ribeirão Preto a taxa aproxima-se das mais elevadas.

No Brasil e em outros paises subdesenvolvidos, a disponibilidade de dados sobre morbidades e atos médicos é bastante deficiente. Com a ausência de taxas de cirurgias para populações específicas, poderíamos apontar alguns coeficientes de internações em cujo total se encontram incluidas as internaçōes cirúrgicas. $O$ coeficiente de internação no Brasil, em 1973, foi de 68 por 1000 habitantes; apresentava-se bastante desigual entre regióes e Estados do país: São Paulo, 103; Rio de Janeiro, 90; Sul, 85; Centro-Oeste, 58; Norte, 44; Maranhão e Piauí, 19 (Mello ${ }^{15}, 1981$ ).

Os coeficientes de internação encontrados em países subdesenvolvidos são, como esperado, inferiores aos observados nos países mais industrializados. Em 1968, o Chile apresentou uma taxa de 90, a Guyana, 82, El Salvador, 51, e Trinidad Tobago, 88, sendo que em Ribeirão Preto, em 1973, o coeficiente de internação foi de 122 por 1000 habitantes (Yazlle Rocha 24, 1975).

Verifica-se, desta forma, que a taxa de internação de Ribeirão Preto é mais elevada que as observadas em paises da América Latina e em outras regiões brasileiras. Em 
EARROS. M.B. de A. A incidência de cirurgias na população de Ribeirão Preto. SP, Brasil. Rev. Saúde públ., S. Paulo, 16:268-81, 1982.

T A B E L A 1

Taxas de cirurgia (por mil habitantes), segundo o sexo do paciente, excluidas as cirurgias obstétricas.

\begin{tabular}{lccc|c}
\hline & & L oc a l & & \\
\cline { 2 - 5 } & Liyerpool & Upusala & New England & $\begin{array}{c}\text { Ribeirão } \\
\text { Preto } \\
(\mathbf{1 9 7 5})\end{array}$ \\
\hline Masculino & 30,0 & 38,1 & 48,7 & 43,7 \\
Feminino & 29,9 & 40,5 & $\mathbf{5 6 , 9}$ & $\mathbf{4 1 , 2}$ \\
\hline
\end{tabular}

Fonte: Pearsun e col. (1968).

T A B E L A 2

Taxas de cirurgia (por mil habitantes) de alguns paises.

Checosloraquia

Inglaterra e Gales

Escócia.

Israel

Itália

EUA

Fonte: Logan e Eimerl (1965).

1975, a taxa de internação de Ribeirão Preto foi de 131,9 , sendo que sua taxa de cirurgia nesse ano $(62 / 1000)$ aproximou-se da taxa global de internações do Brasil (68/1000 en 1973), o que evidencia os elevados coeficientes de internação e cirurgia do municipio estudado.

Quando observada a realização de cirurgias entre as pessoas hospitalizadas obtém-se um indice de 43,7 intervenções por 100 hospitalizações que, desagregado por sexo a grupo etárjo, revela-se maior nos pacientes mais jovens, reduzindo-se após os 45 anos (Fig. 2). A proporção de internações cirúrgricas em Ribeirão Preto é superior às observadas por Pearson e col.17 (1968), ou seja, em Liverpool $(41 \%)$ e Uppsala $(35 \%)$, sendo inferior apenas à de New England (49\%).

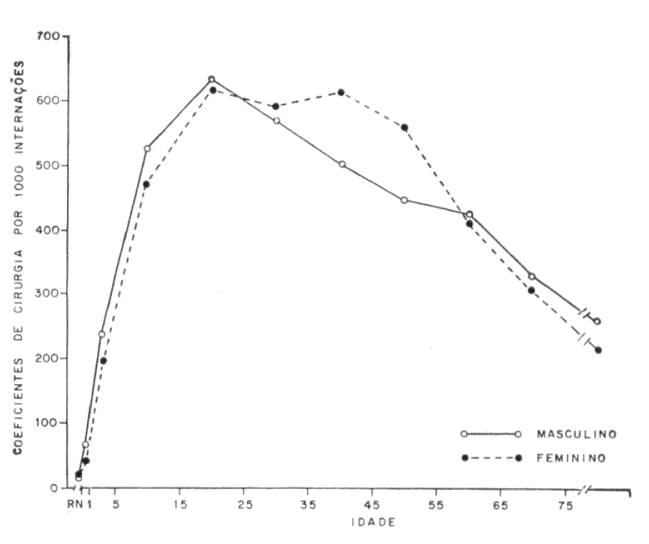

Fla. 2 - Cirurgias de pacientes procedentes de Ribeirão Preto, segundo sexo e grupo etário. Hospitais Gerais de Ribeirão Preto - 1975.

As intervençoes obstétricas representaram $31,8 \%$ do total de cirurgias realizadas, sendo que os procedimentos ortopédicos foram os mais freqüentes no sexo masculino $(23,4 \%)$.

Houve uma diferença acentuada em relação aos sexos quanto ao tipo de interrenção cirúrgica realizada, sendo interessante a observação de taxas mais elevadas, no sexo masculino, de cirurgias ortopédicas, abdominais e plásticas (Fig. 3).

A comparação da incidência de cirurglas na população de Ribeirão Preto com a observada por alguns autores (Logan e Eimerl 11, 1965; Pearson e col.17 1968 e 
EARRoS, M.B. de A. A incidência de cirurgias na população de Ribeirão Preto, SP, Brasil. Rev. Saúde públ., S. Paulo, 16:268-81, 1982.

Bunker ${ }^{4}$, 1970) revela que as taxas das várias especialidades cirúrgicas são, em geral, mais elevadas que as encontradas em regiões da Europa e inferiores às de localidades dos EUA; no município de Ribeirão Preto foram especialmente elevadas as fre- qüências de cirurgias ortopédicas, de postectomias e hemorroidectomias e bastante baixa a incidência de apendicectomia.

Nas Figs. 4.A e 4.B encontram-se os coeficientes de cirurgia por especialidade, discriminados por sexo e idade, oferecendo

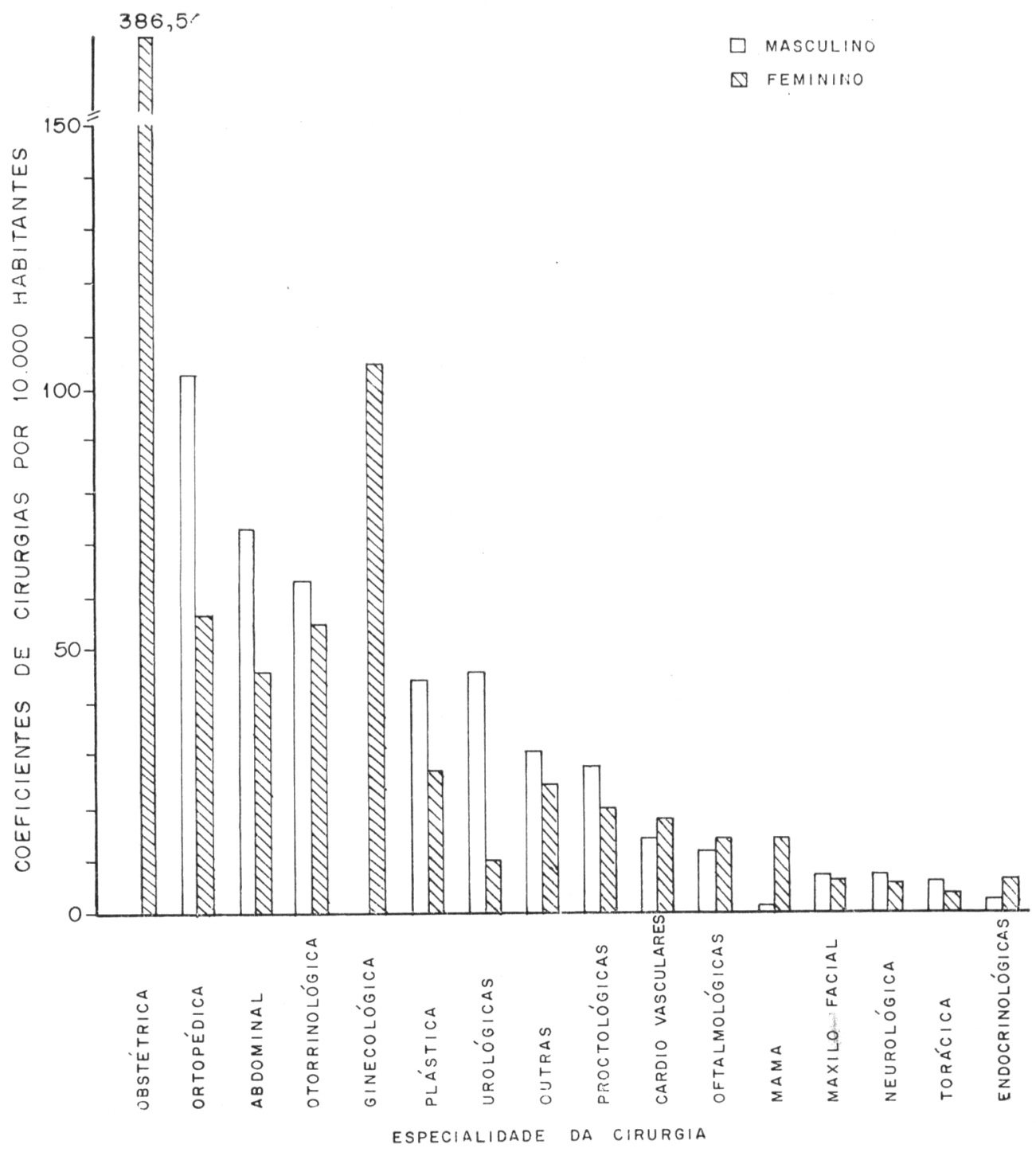

Fig. 3 - Cirurgias realizadas em pacientes procedentes de Ribeirão Preto, segundo sexo e especialidade da cirurgia. Hospitais Gerais de Ribeirão Preto - 1975. 
BARROS, M.B. de A. A incidência de cirurgias na população de Ribeirão Preto, SP, Brasil. Rev. Saúde públ., S. Paulo, 16:268-s1, 1982.
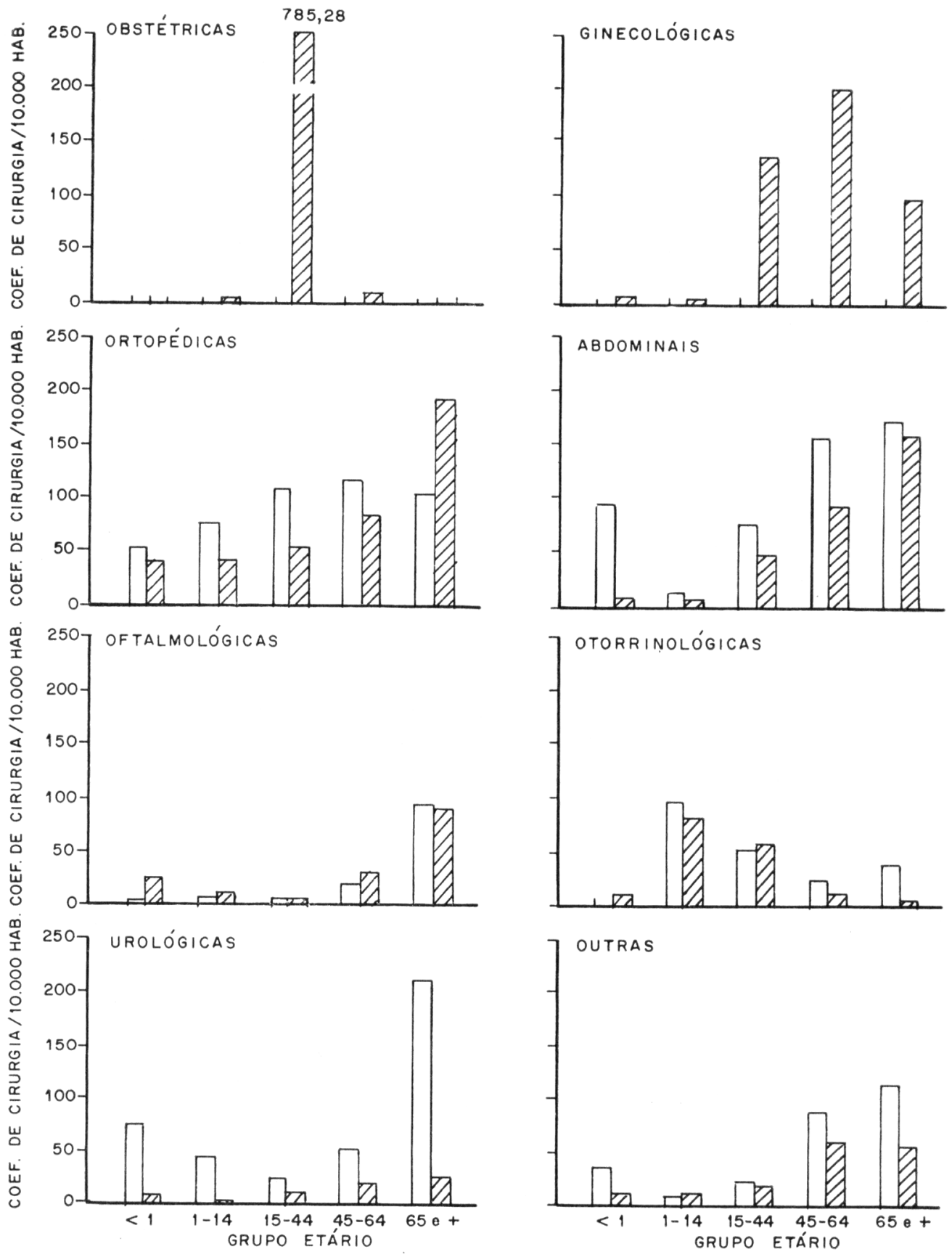

MASCULINO

FEMININO

Fig. 4.A - Cirurgias realizadas em pacientes residentes em Ribeirão Preto, scgundo sexo e grupo etário. Hospitais Gerais de Ribeirâo Preto - 1975.

274 

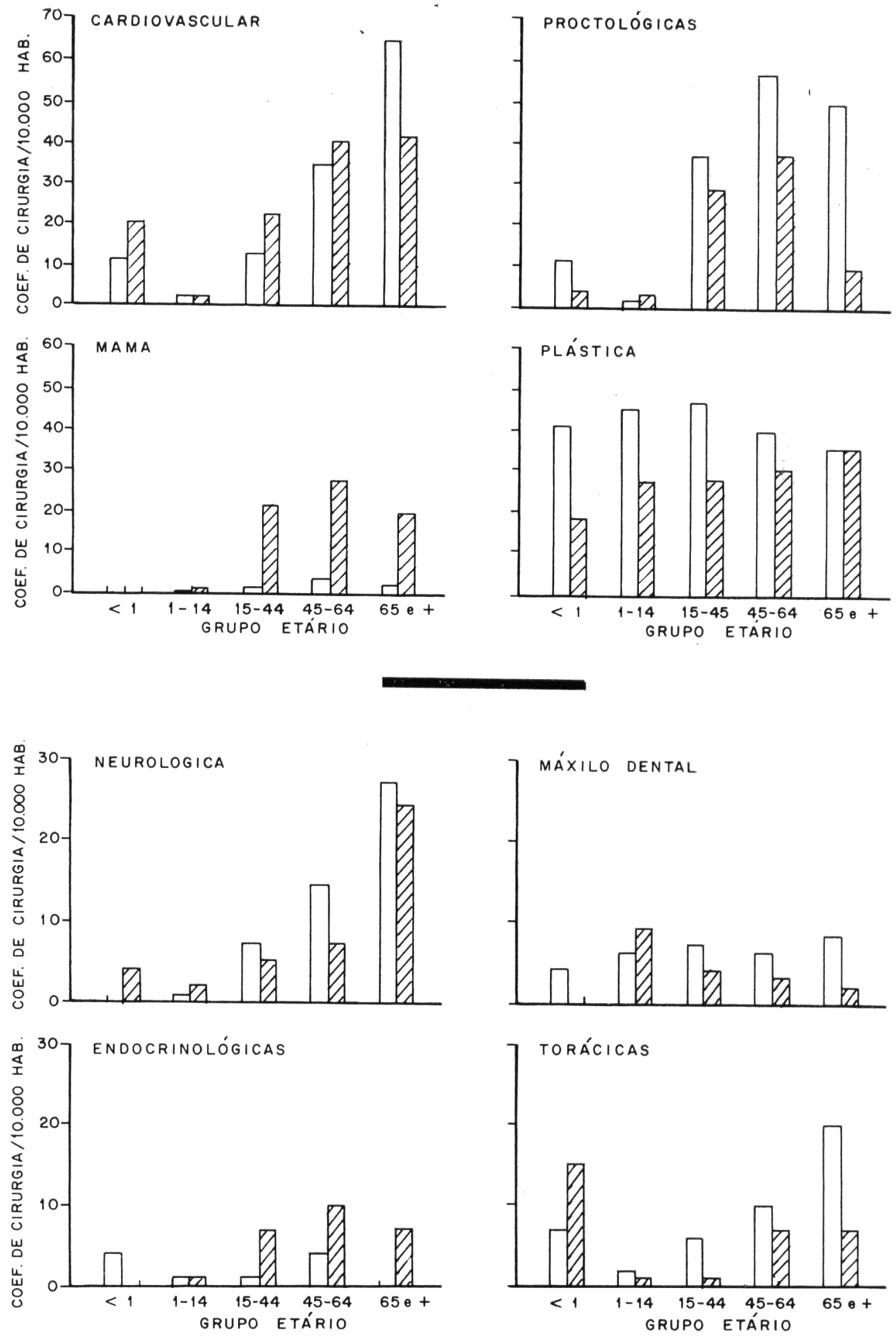

MASCULINO

Q $D E M I N I N O$

Fig, 4.B - Cirurgias realizadas em paciente; residentes em Ribeirão Preto, segundo sexo e grupo etário. Hospitais Gerais de Ribeirão Preto - 1975. 
BARROS, M.B. de A. A incidência de cirurgias na população de Ribeirão Preto, SP, Brasil. Rev. Saúde públ., S. Paulo, 16:268-81, 1982.

uma visão mais detalhada da ocorrência das intervenções.

A elevada taxa de cirurgias da população de Ribeirão Preto acompanha $o$ alto coeficiente de internação verificado nesse município. Evidentemente, trata-se de uma cidade brasileira que, ao concentrar recursos do setor saúde, apresenta-se com uma situação distinta da observada na maioria dos municípios brasileiros, muitos dos quais (aproximadamente 1500) sequer providos da presença de um médico. Entretanto, frente a essa situação de carência geral, merece mais atenção e estudo a intensa produção, ainda que localizada, de serviços de saúde de alto custo e de baixa eficácia na resolução das patologias mais freqüentes.

As intervenções cirúrgicas alén de implicarem atendimento médico mais caro, acarretam riscos maiores para o paciente do que, em geral, os decorrentes de cuidados médicos mais conservadores. Mesmo cirurgias simples encerram riscos importantes; as tonsilectomias, como exemplo, provocavam de 100 a 300 mortes por ano, nos EUA, além da considerável incidência de complicações sérias (Bakwin ², 1958).

Por outro lado, os critérios de indicação de várias intervenções cirúrgicas persistem susceptiveis a questionamentos. Ainda em 1973, nos EUA, Logerfo e col.12 (1978), em uma auditoria médica, verificaram que aproximadamente $67 \%$ das tonsilectomias e/ou adenoidectomias poderiam ou deveriam ter sido evitadas, o que levaria a uma redução de 445.000 intervenções, representando uma economia de 183 milhöes de dólares, naquele ano.

Bolande: (1969) considera, inclusive, que as tonsilectomias e postectomias não se assentam, na maior parte das vezes em que são realizadas, em bases científicas e racionais.

A variabilidade das taxas de cirurgias não tem encontrado explicação suficiente nas diferenças de perfis de morbidade, revelando a existência de outros critérios nem sempre claros e inteiramente justificáveis, de um ponto de vista médico, para a realização de várias intervenções cirúrgicas. A ocorrência de cirurgias desnecessárias tem sido detectada, em alguns países, por auditorias médicas e também pela observação de que tende a ocorrer uma redução da freqüência de cirurgias nos hospitais que foram submetidos a estudos, 'após a divulgação e discussão das taxas encontradas. Foi o que verificaram Dyck e col. ${ }^{\tau}$ (1977) após um estudo sobre a ocorrência de histerectomias em Saskatchewam, e também Wennberg e col. ${ }^{25}$ (1977) após uma pesquisa sobre incidência de tonsilectomias em Vermont.

\section{Cirurgia e modalidade de atendimento médico}

O sistema previdenciário custeou 60,96\% das cirurgias realizadas em Ribeirão Preto, em 1975, em pacientes procedentes deste municipio; a medicina de caridade e a exercida no hospital universitário responderam por $22,73 \%$ delas. Em um esquema de atenção médica liberal foram realizadas $7,38 \%$ das cirurgias e $8,93 \%$ por outros seguros.

Considerando-se o coeficiente de cirurgia por 1.000 internações, verificou-se que 0 maior foi encontrado na categoria de pacientes particulares, em ambos os sexos (Fig. 5). Os indigentes e beneficiários do INPS apresentaram as menores proporções de internações cirúrgicas, sendo que, no sexo masculino, enquanto o menor valor foi encontrado nos indigentes, os segurados do INPS apresentaram um coeficiente relativamente alto, inferior apenas ao dos pacientes particulares. A menor proporção de internações cirúrgicas foi encontrada nos indigentes do sexo masculino.

A observação dos cinco grupos de cirurgias mais freqüentes, em relação à modalidade de atendimento médico, revelou algumas diferenças importantes (Tabela 3). No sexo masculino, as intervençōes ortopédicas foram proporcionalmente mais freqüentes nos pacientes do INPS, outros seguros e indigentes, tendo representado apenas $13 \%$ 
BARROS, M.B. de A. A incidência de cirurgias na população de Ribeirão Preto, SP, Brasil, Rev. Saúde pribl., S. Paulo, 16:268-81, 1982.

T A B E L A 3

Especialidades cirúrgicas por ordem decrescente de freqủencias, segundo a categoria de internação. Hospitais Gerais de Ribeirão Preto - 1975.

SEXO MASCULINO

\begin{tabular}{|c|c|c|c|c|c|c|c|}
\hline \multicolumn{2}{|l|}{ Particular } & \multicolumn{2}{|c|}{ Indigente } & \multicolumn{2}{|l|}{ I N P S } & \multicolumn{2}{|c|}{ Outros seguros } \\
\hline & $N^{\varphi}$ & & $\mathrm{Nu}^{0}$ & & Nu & & $\mathrm{N}$ \\
\hline Otorrinológicas & $\begin{array}{l}s \quad 82 \\
(20,55)\end{array}$ & Abdominais & $\begin{array}{r}144 \\
(17,65)\end{array}$ & Ortopédicas & $\begin{array}{r}967 \\
(26,49)\end{array}$ & Ortopédicas & $\begin{array}{r}85 \\
(20,83)\end{array}$ \\
\hline Urológicas & $\begin{array}{r}72 \\
(18,05)\end{array}$ & Ortopédicas & $\begin{array}{r}142 \\
(17,40)\end{array}$ & Abdominais & $\begin{array}{r}607 \\
(16,63)\end{array}$ & Abdominais & $\begin{array}{r}74 \\
(18,14)\end{array}$ \\
\hline Abdominais & $\begin{array}{r}63 \\
(15,79)\end{array}$ & Urológicas & $\begin{array}{r}132 \\
(16.18)\end{array}$ & Otorrinológicas & $\begin{array}{l}520 \\
(14,24)\end{array}$ & Urológicas & $\begin{array}{r}71 \\
(17,40)\end{array}$ \\
\hline Ortopédicas & $\begin{array}{r}52 \\
(13.03)\end{array}$ & Otorrinológica & $\begin{array}{l}100 \\
(12,25)\end{array}$ & Plástica & $\begin{array}{r}430 \\
(11,78)\end{array}$ & Otorrinológica & $\begin{array}{l}\text { as } 56 \\
(13,73)\end{array}$ \\
\hline Proctológicas & $\begin{array}{r}31 \\
(7,77)\end{array}$ & Plástica & $\begin{array}{r}59 \\
(7,23)\end{array}$ & Urológicas & $\begin{array}{r}283 \\
(7,75)\end{array}$ & Outras & $\begin{array}{r}25 \\
(6,13)\end{array}$ \\
\hline
\end{tabular}

SEXO FENININO

\begin{tabular}{|c|c|c|c|c|c|c|}
\hline \multicolumn{2}{|c|}{ Particular } & \multicolumn{2}{|c|}{ Indigente } & I N $\mathbf{P}$ & \multicolumn{2}{|c|}{ Outros seguros } \\
\hline & $\mathrm{N}^{\varphi}$ & & No & No & & No \\
\hline Obstétricas & $\begin{array}{c}237 \\
(32.07)^{*}\end{array}$ & Obstétricas & $\begin{array}{r}1593 \\
(59,29)\end{array}$ & $\begin{array}{r}2732 \\
(47,27)\end{array}$ & Obstétricas & $\begin{array}{r}339 \\
(35,02)\end{array}$ \\
\hline Ginecológicas & $\begin{array}{r}139 \\
(18,81)\end{array}$ & Ginecológicas & $\begin{array}{r}305 \\
(11,35)\end{array}$ & 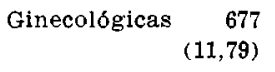 & Ginecológicas & $\begin{array}{r}206 \\
(21,28)\end{array}$ \\
\hline Otorrinológicas & is $\begin{array}{r}64 \\
(8,66)\end{array}$ & Ortopédicas & $\begin{array}{r}128 \\
(4,76)\end{array}$ & Ortopédicas & Abdominais & $\begin{array}{r}80 \\
(8,26)\end{array}$ \\
\hline Abdominais & $\begin{array}{r}55 \\
(7,44)\end{array}$ & Abdominais & $\begin{array}{r}112 \\
(4,17)\end{array}$ & $\begin{array}{r}\text { Otorrinológicas } 488 \\
(8,50)\end{array}$ & Ortopédicas & $\begin{array}{r}69 \\
(7,13)\end{array}$ \\
\hline $\begin{array}{l}\text { Cardiov, e } \\
\text { Ortopédicas }\end{array}$ & $\begin{array}{r}41 \\
(5,55)\end{array}$ & Outras & $\begin{array}{r}107 \\
(3,98)\end{array}$ & Abdominais & Otorrinológicas & $\begin{array}{l}\text { as } \quad 68 \\
(7,02)\end{array}$ \\
\hline
\end{tabular}

* Percentagem em relação ao total de cirurgia do sexo e categoria da internação considerados.

das cirurgias realizadas nos pacientes particulares. Os procedimentos cirúrgicos abdominais ocorreram também, em menor freqüência, nos pacientes da categoria particular.

Sucedeu o contrário com as intervenções otorrinológicas $(60 \%$ de amigdalectomias $\mathrm{e} / \mathrm{ou}$ adenoidectomias) e urológicas $(22 \%$ de postectomias e $16 \%$ de prostatectomias), que foram proporcionalmente mais freqüentes nos pacientes particulares. Cabe ainda assinalar que as cirurgias plásticas corresponderam a $11,8 \%$ das cirurgias realizadas pelo INPS, em pacientes do sexo masculino, e a $7,23 \%$ das realizadas em indigentes, não aparecendo, nas outras categorias de internação, entre as cinco cirurgias mais freqüentes. 


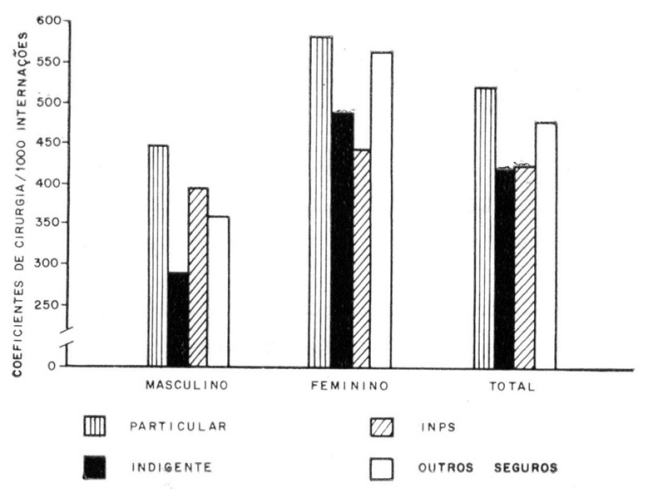

Fig. 5 - Cirurgias realizadas em pacientes procedentes de Ribeirão Preto, segundo sexo e categoria de internação. Hospitais Gerais de Ribeirão Preto -1975 .

No sexo feminino as intervençoes obstétricas e ginecológicas foram as mais freqüentes em todas as categorias, embora com proporçōes muito diferentes e mostraram-se elevadas nos indigentes. As observações feitas quanto às cirurgias ortopédicas e otorrinológicas, no sexo masculino, verificam-se também neste sexo, ocorrendo as primeiras em maior proporção nos pacientes do INPS, de outros seguros e nos indigentes, e as segundas nos pacientes particulares.

Constata-se o papel relevante desempenhado pela Previdência Social, na definição na natureza e tipo da assistência médica existente, quando se verifica que apenas $7 \%$ das cirurgias foram pagas diretamente pelo paciente.

Por outro lado, verifica-se que parcela maior das internações dos pacientes particulares e de "outros seguros" ocorrem com a realização de cirurgias. Como uma parte considerável das cirurgias, principalmente no grupo dos pacientes particulares, constitui-se de procedimentos eletivos, esta observação evidencia a qualidade diferente de atenção médica que é oferecida a segmentos distintos da população. Pode indicar, também, uma "inflação" de internações nos pacientes da previdência social.

A observação dos tipos de cirurgias mais freqüentes, nas quatro categorias de internação consideradas, precisa ser analisada com cautela em função da possível existência de distorções nos dados: ocorrência de cirurgias desnecessárias, de pseudo-cirurgias, alterações nos registros quanto ao tipo de cirurgia realizada, possivel seleção pelos pacientes quanto à categoria de internação em que vão se integrar, em função do tipo e custo da cirurgia a que irāo se submeter.

A maior proporção de intervenções ortopédicas e plásticas nos pacientes do INPS, principalmente no sexo masculino, parece relacionada ao tipo de morbidade que afeta os trabalhadores, ao seu alto grau de exposição a riscos, especialmente à ocorrência de acidentes de trabalho, que apresentavam taxas crescentes no Brasil, na década de 70, atingindo o valor oficial máximo em 1975 (Tambellini Arouca 22, 1978).

Além disso, a diferença existente nas proporções de internações cirúrgicas entre o sexo masculino e feminino dos pacientes da previdência, assim como a já referida maior frequêencia de cirurgias ortopédicas e plásticas, estariam relacionadas ao papel do INPS de reparação física da força de trabalho. Convém lembrar que a taxa de intervençōes ortopédicas encontrada em Ribeirão Preto, foi mais elevada que a observada em paises desenvolvidos, como referido anteriormente.

Assim, o estudo dos tipos de cirurgias realizadas nas diferentes categorias de internação refletem, por um lado, o padrão de morbidade que prevalece nos diversos grupos sociais e, por outro, as características e objetivos das próprias entidades de assistência médica.

Os achados de maior incidencia de cesáreas em pacientes de melhor nível sócioeconômico e nos regimes de livre escolha 
EARROS, M.B. de A. A incidencia de cirurgias na população de Ribeirão Preto, SF, Brasil. Rer. Saíde pübl., S. Paulo, 16:268-81, 1982.

e pagamento por unidade de serviço (Carvalheiro ": 1975; Mello ${ }^{1+}$, 1977) revelam também a interferência de fatores sociais na indicação de cirurgias.

Entretanto, tem sido denunciadas situaçĩes mais graves que divergências relativas a critérios de indicação cirúrgica (Mello ${ }^{14}$, 1977). Inclusive, o ex-presidente do INAMPS, declarou que 600 mil internações desnecessárias teriam sido realizadas na rede contratada da Previdencia Social, em 1976 (Mello 1.5, 1981).

O consumo de intervenções cirúrgicas, referente ao padrão de morbidade prevalente no pais, merece ser analisado detidamente por autores brasileiros. A atuação mais racional do setor médico-hospitalar envolve desde mudanças na formação do médico e do cirurgião, como analisado por Cope" (1965), até alterações necessárias da Politica Nacional de Saúde, de modo a torná-la mais condizente com as necessidades globais de saúde do país. As auditorias médicas e a divulgação das taxas de cirurgias, realizadas por diferentes entidades hospitalares, poderiam contribuir para yue as indicaçoes se tornassem cada vez mais apropriadas. Seria útil a criação, também no que se refere a serviços de saúde, de órgãos de defesa do consumidor brasileiro.

O estabelecimento de medidas efetivas para um controle adequado das intervençoes cirúrgicas poderia evitar que se chegasse a medidas drásticas de suspensão de cirurgias eletivas (portaria 1.265 do Ministro da Previdência Social - Diário Oficial de 18/09/81), em momentos de grave crise financeira da Previdência Social.

\section{CONCLUSÕES}

1. Observou-se elevado coeficiente de cirurgia na população residente no municipio de Ribeirão Preto, em 1975 (62,24/1000 habitantes). O coeficiente foi maior no sexo feminino $(79,82 / 1000)$, especialmente no periodo reprodutivo.

2. As cirurgias obstétricas representaram $31,8 \%$ do total de intervenções realizadas naquele ano. No sexo masculino foram mais freqüentes as cirurgias ortopédicas, abdominais e otorrinológicas.

3. As taxas de intervenções cirúrgicas aumentam com a idade no sexo masculino. enquanto que as internaçōes cirúrgicas. em relação ao conjunto das internações realizadas, decrescem a partir dos 25 anos.

4. A Previdência Social custeou $60,9 \%$ das operações, enquanto que num esquema de medicina liberal foram feitas apenas $7,38 \%$, o que indica o papel fundamental que aquela instituição desempenha na determinação da natureza e intensidade dos serviços médicos produzidos.

5. Os pacientes particulares apresentaram maior coeficiente de cirurgia por internação, sendo mais freqüentes nesta categoria as cirurgias otorrinológicas e urológicas constituídas, em grande parte, por procedimentos eletivos.

6. Os beneficiários da Previdência que apresentam menor taxa de cirurgias por internaçāo são proporcionalmente mais submetidos a intervenções ortopédicas e plásticas, o que provavelmente se relaciona à elevada exposição a riscos de acidentes a que está submetido o trabalhador e ao papel da assistência médica previdenciária como restauradora da força de trabalho. 
BARROS, M.B. de A. A incídência de cirurgias na população de Ribeirão Preto, SP, Brasil. Rev. Saude puibl., S. Paulo, 16:268-81, 1982.

BARROS, M. B. de A. [Incidence of surgery in Ribeirāo Pretr, SP, Brazil]. Rev. Saúde públ.. S. Paulo, 16:268-81, 1982

ABSTRACT: This paper reports on surgical rates as related to patient's ages sex and categories of hospitalization in the population of Ribeirão Preto, S. Paulo, Brazil. Data were obtained from a hospital information center. The surgical rate in Ribeirão Preto was similar to the highest rates reported in literature. Obstetric surgery accounted for $31.8 \%$ of all operations performed. Orthopedic operations were the most frequent kind of surgery performed on the male sex. Hospitalization with surgery was more frequent in the patients whose care was remunerated. The number of orthopedic and plastic surgical operations was greater in the case of Social Insurance and indigent patients, whereas otorrinological anci urinary tract operations were more frequent in the case of paying patients.

UNITERMS: Surgery, incidence. Hospitalization, Ribeirão Preto, SP.

\section{REFERENCIAS BIBLIOGRÁFICAS}

1. ANDERSON, O.W. \& ANDERSEN, R.M Patterns of use of health services in hanabook of medical sociology. Englewood-Cliffs, N.J.. Prentice-Hall, 1972. p. 386-406.

2. BAKWIN, H. The tonsil-adenoidectomy enigma. J. Pediat., $52: 339-61,1958$

3. BOLANDE, R.P. Ritualistic surgery: circuncision and tonsilectomy. New Engt. J. Med., 280:591-6. 1969.

4. BUNKER, J.P. Surgical manpower: a comparison of operations and surgeons in the United States and in England and Wales. New Engl. J. Med., 282:135$-44,1970$.

5. CARVALHEIRO, C.D.G. Estudo de algumas variáveis associados à populaçăo feminina em idade fértill. Ribeirão Preto, 1975. [Tese de Livre-Docência - Faculdade de Medicina de Ribeirão Preto da USP].

6. COPE, $O$. Unnecessary surgery and technical competence: irreconcilables in the graduate training of the surgeon. Amer. $J$. Surg., 110:119-23, 1965.

7. DYCK, F.J. et al, Effect of survellance on the number of hysterectomies in the province of Saskatchewan. New Engl. $J$. Med., 296:1326-8. 1977.

8. FÁVERO, M. et al. Organização de um centro de informática hospitalar em nível local. Rev. pant. Hosp., 21:151-7, 1973.
9. LEMBCKE, P.A. Measuring the quality of medical care through vital statistics based on hospital service areas. 1. Comparative study of appendectomy rates. Amer, J. publ. Hlth, 42:276-86. 1952.

10. LEWIS, C.E. Variations in the incidence of surgery. New Engl. J. Med., 281:880-4 1969.

11. LOGAN, R.F.L. \& EIMERL, T.S. Case loads in hospital and general practice in several countries. In: Acheson, R. M. Comparability in international epidemiology. New York, Milbank Memorial Foundation, 1965. p. 302-10.

12. LOGERFO, J.P. et al. Tonsillectomtes. adenoidectomies, audits: have surgical indications been met? Med. Care, $16: 950-5.1978$.

13. LOGERFO, J.P. et al, Rates of surgical care in Prepaid Group Practices and the independent setting - What are the reasons for the differences? Med. Care, 17: $1-10,1979$.

14. MELLO, C.G. Saûde e assisténcia médica no Brasil. São Paulo, CEBES/HUCITEC. 1977.

15. MELLO, C.G. O sistema de saúde em crise São Paulo, CEBES/HUCITEC. 1981.

16. ORGANIZACION PANAMERICANA DE LA SALUD. Classificación de operaciones quirurgicas $y$ de otros procedimientos terapeuticos y diagnosticos. Washington. D.C., 1971. (Publ. cient.. 230). 
BARRos, M.B. de A. A incidência de cirurgias na população de Ribeirão Preto, SP, Brasil, Rev. Saúde públ., S. Paulo, 16:268-81, 1982.

17. PEARSON, J.C. et al. Hospital caseloads in Liverpool, New England and Uppsala. Lancet, 2:559-66, 1968.

18. PERROT, G.S. Utilization of hospital services. Amer. J. publ. Hlth, 56:57-64, 1966.

19. PETERSON, O.L. et al. What is value for money in medical care? Lancet, $1: 771-6$, 1967.

20. SALKEVER, D.S. Economic class and differential access to care: comparisons among health care systems. Int. J. Hith Serv., 5:373-96, 1975.

21. STOCKWELL, H. \& VAYDA, E. Variations in surgery in Ontario. Med. Care, 17: 390-6. 1979.

22. TAMBELLINI AROUCA, A. O trabalho e a doença. In: Guimarães, R. Saúde $e$ medicina no Brasil: contribuicão para um debate. Rio de Janeiro, Graal, 1978. p. 93-119.
23. VAYDA, E. A comparison of surgical rates in Canada and in England and Wales. New Engl. J. Med. 289:1224-9, 1973.

24. YAZLLE ROCHA, J.S. Utilização de leitos hospitalares gerals em Ribeirão Preto, São Paulo (Brasil). Rev. Saúde públ., S. Paulo, 9:477-93, 1975.

25. WENNBERG, J.E. et al. Changes in tonsillectomy rates associated with feedback and review. Pediatrics, 59:821-6, 1977.

26. WHITE, E.L. A graphic presentation on age and income differentials in selected aspects of morbidity, disability and utilization of health services. Inquiry, 5:18-30, 1968,

27. WHITE, K.L. et al. International comparisons of medical care utilization. New Engl. J. Med., 277:516-22, 1967.

Recebido para publicação em 30/11/1981

A provado para publicação em 25/08/1982 